\title{
Adaptación y validación de la Escala de Satisfacción con el Apoyo Recibido de la Pareja y diseño y validación de la Escala de Disposición a Recibir Apoyo Social
}

\section{Adaptation and Validation of the Satisfaction with Support Received from Partner Scale and the Design and Validation of the Willingness to Receive Social Support Scale}

\author{
Rozzana Sánchez-Aragón, \\ Nazira Calleja \\ Universidad Nacional Autónoma de México, ciudad \\ de México, México \\ Universidad Nacional Autónoma de México, ciudad \\ de México, México
}

\section{Resumen}

La relación de pareja es uno de los mejores escenarios en donde el apoyo se manifiesta como recurso a la vez que como estrategia de mantenimiento y satisfacción entre sus miembros; sin embargo, su operacionalización ha sido parcialmente abordada y no se ha estudiado la disposición a recibirlo y la satisfacción con su recepción. Por ello, esta investigación se propuso adaptar y validar el Cuestionario de Apoyo Social (CAS) de Sarason et al. (1983) en el contexto de la relación de pareja con el propósito de diseñar y validar una escala de disposición para recibir apoyo social ambas en México, así como evaluar la invarianza de medición por sexo para ambas escalas. Tras contar con la participación de 202 parejas heterosexuales mexicanas, se realizaron análisis factoriales exploratorios y confirmatorios con el fin de examinar tanto la conformación factorial como el ajuste al modelo y las posibles variaciones por sexo en cada caso. La Escala de Satisfacción con el Apoyo Recibido de la Pareja (antes CAS) mostró tres factores, lo cual indica especificidades no reportadas previamente, mientras que la Escala de Disposición a Recibir Apoyo Social resultó unidimensional. Con base en esto, se obtuvieron dos escalas cuyos índices de confiabilidad y validez resultaron satisfactorios para su uso en investigación básica y aplicada.

Palabras clave: apoyo, pareja, medición, validación, México.

Rozzana Sánchez-Aragón, profesora Titular C Tiempo Completo Definitiva, Universidad Nacional Autónoma de México, México.

Nazira Calleja, Investigadora Titular C Medio Tiempo Definitiva, Universidad Nacional Autónoma de México, México.

La correspondencia en relación con este artículo se dirige a Rozzana Sánchez-Aragón, Universidad Nacional Autónoma de México. Dirección electrónica: rozzara@unam.mx 


\title{
R. SÁNCHeZ y N. CALLEJa
}

\begin{abstract}
:
A couple's relationship is one of the best scenarios where support manifests itself as a resource as well as a maintenance and satisfaction strategy between its members, nevertheless, its operationalization has been partially addressed but the willingness to receive and satisfaction with the support provided haven't been studied. Therefore, this research was proposed to adapt and validate the Sarason et al. (1983) Social Support Questionnaire (SSQ) in the context of the couple's relationship, to design and validate a measure of willingness to receive social support, both in Mexico, and evaluate the measurement invariance by sex for both scales. With the participation of 202 Mexican heterosexual couples, exploratory and confirmatory factor analyses were carried out in order to examine both the factorial conformation and the fit to the model and the possible variations by sex in each case. The measure of Satisfaction with Support received from Partner (formerly SSQ) provided three factors, which indicate specificities not previously reported, while the measure of willingness to receive social support was one-dimensional. Based on this, two measures were obtained whose reliability and validity indices were satisfactory for use in basic and applied research.
\end{abstract}

Keywords: Support, Couple Relationships, Measurement, Validation, Mexico.

Durante el proceso de vida de una pareja, se presentan en forma natural eventos de desafío que conllevan periodos de estrés agudo o crónico para uno o ambos miembros de la pareja; por ejemplo: comprar una casa, tener problemas financieros, volverse padres, enfrentar la enfermedad de los propios padres o completar un proyecto importante. Este tipo de situaciones han mostrado ejercer un impacto poderoso y negativo en los individuos y en las diadas (Bodenmann, 2005; Karney et al., 2005), particularmente, porque sus miembros pueden percibirse incapaces o abrumados al comparar los recursos que poseen con la magnitud de la amenaza percibida (Flórez-Rodríguez \& Sánchez-Aragón, 2019).

Ante una situación de estrés, es factible que cada miembro de la relación de pareja despliegue, por un lado, su capacidad para afrontar individualmente o adaptarse exitosamente a una situación a pesar de la amenaza (Palomar-Lever \& Gómez-Valdez, 2010); y por otro lado, el hecho de disponerse a recibir ayuda externa al desplegar comportamientos facilitadores para que quien pueda se la brinde -p. ej., su pareja. Al respecto, Gurung et al. (1997) señalan que justamente la percepción de que se puede contar con ayuda de la pareja se relaciona con las propias características del receptor, que facilitan que quien se encuentre cerca brinde el apoyo; así, una persona que es sociable, abierta y positiva tenderá a compartir de su vida y estar con ello dispuesta y expuesta a recibir consejo, ayuda y soporte. De esta manera, se puede complementar el proceso interactivo de apoyo (Bodenmann et al., 2008); es decir, unir los propios recursos con aquellos provenientes de su relación más cercana.

Una vez que se generó la respuesta de apoyo de un miembro hacia el otro, el estrés es aliviado y con ello la diada desarrolla una forma sincronizada o compartida de afrontamiento (Falconier \& Kuhn, 2019; Sánchez-Aragón, 2016). Por lo tanto, puede asegurarse que como parte de las interacciones que una pareja mantiene a través del tiempo, el apoyo representa un elemento transaccional que brinda beneficios (Sprecher \& Felmlee, 1992; Sullivan \& Davila, 2010) a dos niveles: 
Adaptación y validación de la Escala de Satisfacción con el Apoyo Recibido de la Pareja

- Individual, pues produce un estado de ánimo positivo (Procidano \& Walker-Smith, 1997), incremento de su autoestima (y sus sentimientos de aceptación y valoración), mayor bienestar subjetivo (Gable et al., 2006), refuerza la capacidad del receptor de apoyo para resistir y superar frustraciones (Chi et al., 2011), resolver retos (National Scientific Council on the Developing Child, [NSCDC], 2010), y por lo tanto, contribuye a su ajuste psicológico y salud física (Rivas-Diez, 2013; Sarason et al., 1983); en ese sentido, se limita la posibilidad de psicopatología y se convierte en un factor de protección que proporciona recursos psicológicos necesarios para enfrentar el estrés.

- Relacional, porque capitaliza a los individuos (Revenson, 1994); es decir, juega un papel central en el cumplimiento de las necesidades de apego (Greenberg \& Johnson, 1988) al proveerle una percepción subjetiva de que la relación es "segura" y ello favorece interacciones más positivas, recíprocas y percibidas como balanceadas o equitativas (Collins \& Feeney, 2010; Guerrero et al., 2018; Procidano \& Walker-Smith, 1997). Al mismo tiempo, suaviza los efectos de la vida cotidiana como puede ser la rutina, las cuestiones económicas, las dificultades personales y de la propia relación (Chi et al., 2011; Stafford, 2003; Sullivan \& Davila, 2010) y en consecuencia repercutir en la satisfacción que ambos miembros tienen de su relación (Dainton, 2011; Vidal-González et al., 2012).

Ahora bien, el estudio del apoyo en cualquier relación con otra persona (llamado apoyo social), incluyendo a la pareja, se define como la existencia o la disponibilidad de alguien que pueda auxiliar, proteger, respaldar, defender, cuidar, valorar y amar a otra persona (Sarason et al., 1983), cuyas funciones incluyen sustento emocional, desarrollo de la autoestima, provisión de información y asistencia tangible (Cutrona \& Russell, 1987). Se han identificado dos tipos principales: el apoyo instrumental y el apoyo emocional. El primero consiste en la ayuda o la asistencia de una manera tangible o física como proveer dinero, aportar soluciones prácticas o colaborar al realizar una tarea; mientras que el segundo involucra escuchar, ser empático y ofrecer afecto ante eventos de necesidad (Burkert et al., 2005; Morelli et al., 2015). Este constructo ha sido muy popular en la literatura especializada; sin embargo, en México e Iberoamérica su investigación ha sido limitada cuando se trata de estudiarlo en la relación de pareja. Algunos ejemplos de estos esfuerzos lo representan el estudio de Sánchez-Aragón y Díaz-Loving (2002), quienes identificaron un factor de apoyo en el Inventario Conductual de la Pareja que incluye comportamientos como ayudar, apoyar, resolver problemas, dar ánimo, ser comprensivo y escuchar y más recientemente Sánchez-Aragón (2018) encontró un factor de apoyo en su Inventario de Afectividad para la pareja que trata sobre el apoyo que se brinda a la pareja cuando lo necesita mediante la ayuda, el ánimo, la escucha, el cuidado, la compañía, la comprensión y las muestras de interés.

Fuera de México, se han documentado en la literatura otros autorreportes que se diseñaron para medir al apoyo en las relaciones cercanas (familiares, de pareja y de amistad) como las siguientes:

- Cuestionario de Apoyo Social (Sarason et al., 1983) evalúa el número personas que han proveído de apoyo social (hermano, amigo, padre, etc.) y el gusto experimentado con el apoyo que se recibió por medio de 27 reactivos con formato tipo Likert de seis puntos indicando grados de satisfacción. Todos se han concentrado en un solo factor que obtuvo un $\alpha=.94$. Algunos ejemplos de reactivos son los siguientes: “¿Con quién puede usted realmente contar o depender cuando necesita ayuda?”, “¿Con quién puede usted ser usted mismo totalmente?” y “¿Quién le reconfortará cuando necesite un abrazo?”. 


\section{R. SÁNCHez y N. CALLEJa}

- Escala Multidimensional de Apoyo Social Percibido (Zimet et al., 1988) incluye 12 reactivos en formato tipo Likert, de siete opciones de respuesta, distribuidos en tres factores: familia (e.g., "Mi familia realmente trata de ayudarme", $\alpha=.87$ ), amigos (e.g., "Tengo amigos con quienes puedo confiar mis felicidades y tristezas", $\alpha=.85$ ) y otros significativos (e.g., "Hay una persona especial que siempre está cerca cuando la necesito", $\alpha=.91$ ).

- Cuestionario de Interacción y Apoyo Social (Verhofstadt et al., 2007) en el que se les pedía a los participantes que dijeran si discutían con sus parejas sus problemas personales y que estimaran la posibilidad de solicitar/brindar distintos tipos de apoyo durante sus discusiones. Consta de 54 reactivos en formato de respuesta tipo Likert de 9 opciones indicando la posibilidad de pedir o dar apoyo y se agruparon en cinco factores: búsqueda de ayuda positiva (e.g., dar un claro análisis del problema, reconoce a su pareja como ayuda), búsqueda de ayuda negativa (e.g., rechaza ayuda, critica a quien le ayuda), ayuda positiva emocional (p. ej., expresión de cuidado de quien busca ayuda, entendimiento), ayuda positiva instrumental p. ej., ofrece un plan específico o asistencia) y conducta negativa de ayuda (critica, minimiza el problema). En cuanto a su confiabilidad, se reportan Alphas de Cronbach entre .73 y .87 en hombres, .76 y .85 en mujeres.

- Escala de Estimación de Apoyo en las Relaciones Íntimas (Dehle et al., 2001) fue desarrollado para medir la frecuencia con la que se presentan las conductas de apoyo por parte de la pareja al tomar en consideración la adecuación del apoyo (diferencia entre el recibido y el deseado). Responden a la escala - cuyo coeficiente Alpha de Cronbach resultó de .97-durante un periodo de siete días consecutivos para mejorar la estabilidad del autorreporte y las calificaciones finales pueden indicar una carencia de apoyo, apoyo de más, o bien apoyo a la altura de las necesidades.

- Escala de Apoyo Emocional e Instrumental, Burkert et al. (2005) crearon dos instrumentos con dos reactivos en formato tipo Likert de seis puntos de respuesta (no se reportan coeficientes de confiabilidad) en los que preguntaban sobre los actos de apoyo emocional e instrumental recibidos o al brindarlos entre los miembros de la pareja durante la semana anterior relativos a fumar (e.g., "Mi pareja me ha aceptado y ha demostrado que le gusto aun cuando estoy estresado porque no puedo fumar", "Mi pareja me recuerda estrategias que me ayudan a resistir fumar").

- El Cuestionario de Apoyo Recibido de la Ex Pareja (CARE) de Yárnoz-Yaben (2010) está compuesto por ocho ítems agrupados en un solo factor que explica el $41.76 \%$ de la varianza y que cuenta con un $\alpha=.79$. Algunos de sus reactivos son los siguientes: "En general, siento que mi expareja entiende y apoya mis necesidades como progenitor" y "Cuando yo he necesitado hacer un cambio en los acuerdos de visita, mi expareja ha cedido para que pudiera adaptarme mejor".

- Escala de Provisión de Apoyo (Devoldre et al., 2010) es un autorreporte de 54 ítems en formato Likert de nueve opciones de respuesta que evalúan el rol de quien da apoyo y el de recibirlo con base en Bradbury y Pasch (1994). Este cuestionario tiene tres subescalas con $\alpha$ entre .66 y .77, que indican la probabilidad de provisión de apoyo emocional (e.g., entendimiento, proveer de ánimo genuino), instrumental (e.g., dar consejos de ayuda, ofrecer un plan específico de apoyo) y negativo (e.g., criticar, no estar atento).

- Escala de Apoyo de la Pareja (Cienfuegos-Martínez \& Díaz-Loving, 2011) consta de un solo factor que comprende 22 reactivos en formato tipo Likert que indican grados de acuerdo y que poseen un 
Adaptación y Validación de la Escala de Satisfacción con el Apoyo Recibido de la Pareja

$\alpha=.96 \mathrm{y}$ dos indicadores sobre apoyo en el hogar y cuidado de los hijos. Algunos ejemplos de reactivos son los siguientes: "Mi pareja me da alternativas sobre cómo solucionar un problema", "Mi pareja me alienta para enfrentar cualquier adversidad" y "Mi pareja hace todo lo posible para que yo esté bien".

- Escala de Valoración de la Capacidad de Soporte y de Dependencia (Gómez-Zapiain et al., 2012) mide dos dimensiones: Soporte con 9 reactivos y un $\alpha=.82$ (e.g., "Cuando mi pareja está preocupada, disgustada o triste yo me muestro próximo y cercano para darle apoyo y consuelo") y Dependencia con 6 reactivos y un $\alpha=.80$ (p. ej., "Cuando me siento mal, angustiado, deprimido, necesito recurrir urgentemente a mi pareja").

- Tareas de Interacción de Apoyo Social (Verhofstadt \& Weytens, 2013) consta de una serie de 18 escenarios que describen estresores cotidianos relativos a demandas, conflictos interpersonales o tensiones y otros (financieros o de transporte) y ante los cuales los participantes deben indicar como responderían ante la divulgación de estrés de su pareja al seleccionar una de diez respuestas que reflejan tres tipos posibles de apoyo: emocional, instrumental o apoyo inútil (no se reportan datos de confiabilidad).

En general, se observa que la mayor parte de las medidas citadas -excepto la de CienfuegosMartínez y Díaz-Loving (2011)- fueron realizadas y validadas en Estados Unidos, Alemania, España, y Bélgica para otras relaciones cercanas tales como familia y amigos. Aunado a ello, se nota que se evalúan, principalmente, el acuerdo o frecuencia con las conductas de apoyo y una gran variabilidad en cuanto a la constitución del constructo (unidimensional o multidimensional). Si bien algunas de las escalas descritas son aplicadas a contextos particulares (expareja y coparentalidad, conflicto), varias se enfocan en el apoyo percibido, percibido y brindado, percibido y deseado, lo cual resulta en una contribución al área.

Además de lo anterior, una pregunta que puede surgir es si existen diferencias entre hombres y mujeres respecto a la provisión de apoyo, la solicitud de apoyo e incluso el estilo de pedirlo. Al respecto, Pasch et al. (1997) indican que son las mujeres quienes son proveedoras más efectivas de apoyo en comparación con los hombres, son más hábiles en enviar y entender expresiones emocionales no verbales (ver Verhofstadt et al., 2007) y en consecuencia, los hombres reciben más apoyo de sus esposas que a la inversa. La evidencia lleva a concluir que, en las relaciones de pareja, las mujeres reciben menos apoyo y ayuda del que proveen a sus esposos (e.g., Belle, 1982). En cuanto a la solicitud de apoyo, se ha encontrado que son ellas quienes buscan apoyo más frecuentemente -en comparación con los hombres-, y se sienten más cómodas al pedir y recibir la ayuda en comparación a los hombres (e.g., Thoits, 2011). En tanto, cuando se habla del estilo de apoyo, Tannen (1990) reporta que los hombres suelen proveer información, consejo o soluciones a un problema; mientras que las mujeres que proveen más apoyo emocional son más empáticas y afectuosas durante el proceso. Sin embargo, Neff y Karney (2005) no encontraron diferencias en el promedio de apoyo proveído entre los miembros de las parejas, lo que sí notaron es que difieren en el tiempo que transcurre entre la solicitud y el brindar apoyo y al responder los hombres despliegan apoyo y negatividad.

Como se indicó inicialmente, el apoyo en el contexto de las relaciones de pareja confiere beneficios a distintos niveles en quienes lo reciben; no obstante, la información de estas mismas fuentes es paradójica. Por un lado, la disponibilidad de apoyo tiende a reducir el estrés; por otro lado, puede generar 
sentimientos de insuficiencia, endeudamiento e inequidad no intencionales en quien lo recibe (Vangelisti, 2009). Asimismo, hay que considerar si el apoyo recibido corresponde al deseado (aspecto valorado parcialmente por Dehle et al., 2001), porque en la medida en que estos "correspondan" se podrá estimar al apoyo como exitoso. En esta línea, se observó que la aportación de Sarason et al. (1983) posee ítems que muestrean ampliamente al constructo del apoyo aplicable a la relación de pareja, pues al abordarlo desde la satisfacción que un miembro tiene respecto al apoyo recibido, se controlan los riesgos de que aspectos como (a) el momento en el que se proporciona, (b) el tipo o el contenido, (c) el proceso y (d) la reciprocidad (Rafaeli \& Gleason, 2009) no afecten sus consecuencias o su éxito en la relación de pareja.

A partir de la importancia que posee el apoyo social proveniente de la pareja y el papel que juega la disposición del individuo de permitirse recibir dicho apoyo y la necesidad de evaluar en México a estas dos variables al cubrir los aspectos señalados por Rafaeli y Gleason (2009), el presente estudio tuvo por objetivos: (a) adaptar y validar el Cuestionario de Apoyo Social de Sarason et al. (1983) en el contexto de la relación de pareja, (b) diseñar y validar una escala de disposición para recibir apoyo social ambos en México y (c) evaluar la invarianza en la medición por sexo para ambas escalas.

\section{Método}

\section{Participantes}

Se trabajó con una muestra no probabilística de 202 parejas heterosexuales cuyos miembros tenían edades entre 18 y 62 años $(M=37, D E=11.34)$, con escolaridad de secundaria $(18.3 \%)$, preparatoria (30.8\%) y licenciatura (46.5\%) y en una relación de pareja cuya duración oscila entre 5 meses y 41 años $(\mathrm{M}=14$ años, $\mathrm{DE}=9.81)$. El estado civil reportado fue de unión libre $(45.3 \%)$, casados $(51.7 \%)$ y en cuanto al número de hijos hubo una variedad desde 0 hasta 5 (Moda $=2, \mathrm{M}=1.52, \mathrm{DE}=1.24$ ). Los criterios de inclusión de la muestra fueron que ambos miembros de la relación heterosexual fueran adultos, con escolaridad mínima de secundaria, que vivieran juntos y que el tiempo de relación fuera al menos de cinco meses (esto con el fin de que tuvieran cierto tiempo de convivencia en el cual pudieran manifestarse las variables). Cuando no contaban con alguna de las condiciones se prescindió de su participación.

\section{Instrumentos}

La Escala de Satisfacción con el Apoyo Recibido de la Pareja (ESAReP) se basó en el Cuestionario de Apoyo Social (CAS) de Sarason et al. (1983) que evalúa: (a) el número de apoyos sociales percibidos y (b) la satisfacción con el apoyo social disponible por medio de 27 reactivos con formato tipo Likert de seis puntos de respuesta con un $\alpha$ de .97 y agrupados en un factor que explicó el $72 \%$ de la varianza. Un ejemplo de reactivo es: ¿Quién piensa usted que realmente le aprecia como persona? Con las siguientes respuestas: nadie__ 1) ___ 2) ___ _ y así sucesivamente hasta ofrecer nueve opciones de personas posibles que fueran fuente de apoyo. Enseguida, se pregunta: ¿Cuán satisfecho se encuentra con el apoyo recibido? Con respuestas: 1-muy insatisfecho a 6-muy satisfecho.

Los pasos, que se siguieron en el proceso de adaptación de esta escala, fueron los siguientes:

1. Traducir-retraduciry ajustar los reactivos al contexto de la relación de pareja al partir de la siguiente instrucción: "Las siguientes preguntas son respecto al apoyo o ayuda que usted recibe de su pareja. En cada enunciado, por favor, indique con una X cuán satisfecho se encuentra usted con el apoyo que recibe de ella". 
Adaptación y Validación de la Escala de Satisfacción con el Apoyo Recibido de la Pareja

2. Agregar cuatro reactivos que abordaban temas no mencionados en la escala original y que revelan escenarios en donde el apoyo puede ser importante (ver Guerrero et al., 2018) ("Cuando mi pareja me lleva a algún lugar al que necesito ir", "Con el apoyo económico que recibo de mi pareja", "Con la compañía de mi pareja en momentos de necesidad", "Del tiempo que mi pareja me brinda cuando me hace falta") en concordancia con los primeros.

3. Unificar la forma de respuesta a cinco puntos (Likert) que indicaban grados de satisfacción: 1-Totalmente insatisfecho y 5-Totalmente satisfecho.

4. Ya contando con los 31 ítems, estos fueron evaluados por un grupo de 10 jueces (expertos psicólogos en el tema de las relaciones de pareja y con experiencia en diseño de instrumentos de medición psicológica), los cuales señalaron algunos cambios de redacción, que fueron realizados, lo cual resultó útil para que el instrumento contara con validez de contenido y constructo. Algunos ejemplos de reactivos ya corregidos son los siguientes: "Con la capacidad que tiene mi pareja para escucharme cuando necesito hablar", "Con la ayuda que me brinda mi pareja cuando la necesito", "Con los consejos útiles que mi pareja me da para evitar cometer errores".

Escala de Disposición a Recibir Apoyo Social (EDaRAS) fue diseñada a partir de la investigación de Gurung et al. (1997) y al considerar tanto las actitudes como disposiciones conductuales que guían hacia la posibilidad de recibir apoyo como compartir los propios sentimientos, dejarse ayudar, expresar el propio sentir ante una preocupación, pedir ayuda, hablar de los problemas personales a otros, permitir que la familia y amigos brinden ayuda cuando se tienen dificultades y mantener la disposición de recibir consejos ante una situación difícil. Al considerar esto, se diseñaron siete reactivos en formato tipo Likert de cinco puntos que indican frecuencia y que van de 1-Nunca a 5-Siempre (ver apéndice).

\section{Procedimiento}

La aplicación de los instrumentos se llevó a cabo por psicólogos calificados que acudieron a lugares donde se podían encontrar parejas, o bien personas con una relación de pareja actual que cubrieran los criterios de inclusión (plazas comerciales, casas, escuelas, oficinas, centros recreativos y culturales, etc.). En el caso de que se encontraran personas solas, se les preguntaba sobre la posibilidad de que su pareja colaborara también en la investigación. Así, cuando esto estaba arreglado, se acordaba un lugar y tiempo para ello. De esta forma, se solicitó la participación voluntaria, confidencial y anónima de cada miembro de la relación al explicarles que sus datos solo se utilizarían con fines científicos. Quienes participaron de manera explícita indicaron su consentimiento para ello y durante la aplicación de las escalas se respondió inmediatamente a sus dudas y se dejó a su disposición sus resultados personales. Además, se tomaron las consideraciones éticas y en cumplimiento de las reglas establecidas por la Secretaría de Salud en materia de Investigación (2021), la Asociación Americana de Psicología (2020) y el Código Ético del Psicólogo (Sociedad Mexicana de Psicología [SMP], 2009).

\section{Análisis de datos}

Para cumplir con los dos primeros objetivos de esta investigación se utilizaron el paquete estadístico SPSS versión 25 y el SPSS Amos versión 22, que permitieron realizar los siguientes análisis orientados a la validación de las medidas en cuestión (Reyes-Lagunes \& García-y-Barragán, 2008; Cronbach 
\& Meehl, 1955): (a) análisis de frecuencias, (b) $t$ de Student para identificar reactivos que discriminaron, (c) análisis de confiabilidad Alpha de Cronbach y Omega de McDonald, (d) análisis factorial exploratorio (AFE), (e) análisis factorial confirmatorio (AFC) y (f) análisis de confiabilidad Alpha de Cronbach y Omega de MacDonald de los factores obtenidos en el AFC. Para el tercer objetivo, se efectuaron AFC multi-grupo (Vandenberg \& Lance, 2000)․․

\section{Resultados}

En lo que concerniente a la ESAReP, y al seguir con el procedimiento de adaptación y validación mencionado en el método, no se observaron errores de captura o datos perdidos. De forma posterior, se obtuvieron los cuartiles de la suma de todos los reactivos, lo que permitió tener grupos extremos de puntajes que se usaron para aplicar la prueba $t$ de Student para muestras independientes e identificar cuáles reactivos mostraban diferencias estadísticamente significativas entre ellos y, por tanto, que discriminaban.

Los resultados mostraron que los 31 ítems diseñados discriminaron; en consecuencia, se realizó un AFE de ejes principales con rotación oblicua (tipo promax), porque originalmente la escala mostró unidimensionalidad y se seleccionaron solo aquellos que registraron cargas factoriales iguales o superiores a .40 (excepto el reactivo "Con la capacidad que tiene mi pareja de escucharme cuando necesito hablar" cuya carga fue de .350). Dicho análisis evidenció un índice KMO $=.973$ y la Prueba de Esfericidad de Bartlett $=8154.932, \mathrm{gl}=300, p=.001$, que indica la adecuación de la muestra, además de reflejar la existencia de tres factores con valores superiores a 1 que explicaron el $62.05 \%$ de la varianza y con coeficientes de confiabilidad $\alpha=.979$ y $\omega=859$ (ver tabla 1). Cabe señalar que, en este proceso, seis reactivos no obtuvieron cargas suficientemente altas $(<.40)$ para permanecer en su factor.

\footnotetext{
${ }^{1}$ Sucesivamente, se probaron los modelos de invarianza de configuración (no variabilidad de la estructura factorial entre los grupos, con el libre cálculo de cargas factoriales, interceptos y residuales), invarianza métrica (restricción de la estructura factorial y las cargas factoriales), invarianza escalar (restricción adicional de los interceptos), e invarianza estricta (restricción también de los residuales). Para evaluar las diferencias de los modelos, se evaluaron los cambios en CFI y en RMSEA y la significancia del $\Delta \chi^{2}$. Se apoya una fuerte invarianza cuando el $\Delta \mathrm{CFI} \leq 0.01$, el $\Delta \mathrm{RMSEA} \leq 0.015$ y el $\Delta \chi 2, \mathrm{p}>.05$ (Cheung \& Rensvold, 2002).
} 
Adaptación y validación de la Escala de Satisfacción con el Apoyo Recibido de la Pareja

Tabla 1

Análisis Factorial Exploratorio de la ESAReP

Reactivos

Carga factorial

Factor 1. Satisfacción con la comprensión y la confianza

Con la confianza que mi pareja me da para ser totalmente yo mismo(a).

Cuando mi pareja me expresa que me aprecia como persona.

Con la aceptación total por parte de mi pareja, incluyendo mis peores defectos y cualidades.

Cuando mi pareja me escucha de una manera abierta y sin criticarme cuando le hablo de mis sentimientos más profundos.

Con la forma en la que mi pareja me hace sentir que tengo algo positivo para ofrecer a los demás.

Con la confianza que me brinda mi pareja para poder hablar abiertamente y con franqueza.

Con los consejos útiles que mi pareja me da para evitar cometer errores.

Con los consejos de mi pareja cuando necesito mejorar de alguna manera.

Con que mi pareja me cuide sin importar lo que me esté pasando.

Cuando mi pareja me hace sentir que soy una parte importante de su vida.

Con la capacidad que tiene mi pareja de escucharme cuando necesito hablar.

.350

\begin{tabular}{llll}
\hline 11 reactivos & $\alpha=.951 / \omega=.877$ & $\mathrm{M}=4.15$ & $\mathrm{DE}=.7834$
\end{tabular}

Factor 2: Satisfacción con el apoyo instrumental

Con la compañía de mi pareja en momentos de necesidad.

Con el apoyo económico que recibo de mi pareja.

Con la ayuda de mi pareja en un momento de crisis aún cuando esto implique que deje de hacer sus actividades personales.

Con la ayuda que me brinda mi pareja cuando la necesito. 


\section{R. SÁNCHEZ y N. CALleja}

\begin{tabular}{|c|c|}
\hline Reactivos & Carga factorial \\
\hline Cuando mi pareja me lleva a algún lugar al que necesito ir. & .549 \\
\hline Con la ayuda que me daría mi pareja en caso de que fuera despedido(a) de mi trabajo. & .526 \\
\hline Con todo lo que mi pareja hace por mí cuando lo necesito. & .452 \\
\hline Con el apoyo que mi pareja me da (diera) si algún miembro cercano de mi familia muriera. & .424 \\
\hline Con la ayuda de mi pareja en caso de que yo viviera una situación de duelo. & .412 \\
\hline$\alpha=.930 / \omega=.798$ & \\
\hline
\end{tabular}

Factor 3. Satisfacción con el apoyo emocional

Con la ayuda que me da mi pareja para sentirme mejor cuando estoy muy irritable.

Cuando mi pareja me consuela si estoy muy enojado(a).

Con la ayuda que me brinda mi pareja para sentirme mejor cuando mi ánimo está por los suelos.

Del tiempo que mi pareja me brinda cuando me hace falta.

Con la capacidad que tiene mi pareja para hacerme sentir más relajado cuando estoy bajo presión/tensión.

\begin{tabular}{llll}
\hline 5 reactivos & $\alpha=.922 / \omega=.840$ & $\mathrm{M}=4.05$ & $\mathrm{DE}=.8481$
\end{tabular}

Con el fin de confirmar la estructura factorial de la escala obtenida previamente, se procedió a realizar el AFC, en donde se obtuvo nuevamente un modelo de tres factores (ver figura 1, se muestran las cargas factoriales estandarizadas), lo que significa que se confirma el modelo excepto por cuatro reactivos (uno del primer factor y tres del segundo), que fueron eliminados por presentar altos puntajes residuales. Para determinar el ajuste del modelo propuesto, se analizaron los siguientes índices (Hu \& Bentler, 1999), que resultaron satisfactorios: $\chi^{2}(178)=419.323, \mathrm{CMIN} / \mathrm{DF}=2.356, \mathrm{NFI}=.946, \mathrm{CFI}=$ $.968, \mathrm{AGFI}=.883, \mathrm{SRMR}=.025, \mathrm{RMSEA}=.058(.051-.065)$. Cabe señalar que se observaron altas correlaciones entre los factores que componen a la escala (ver figura 1). 
Figura 1

\section{Análisis Factorial Confirmatorio del modelo de tres factores de la ESAReP}

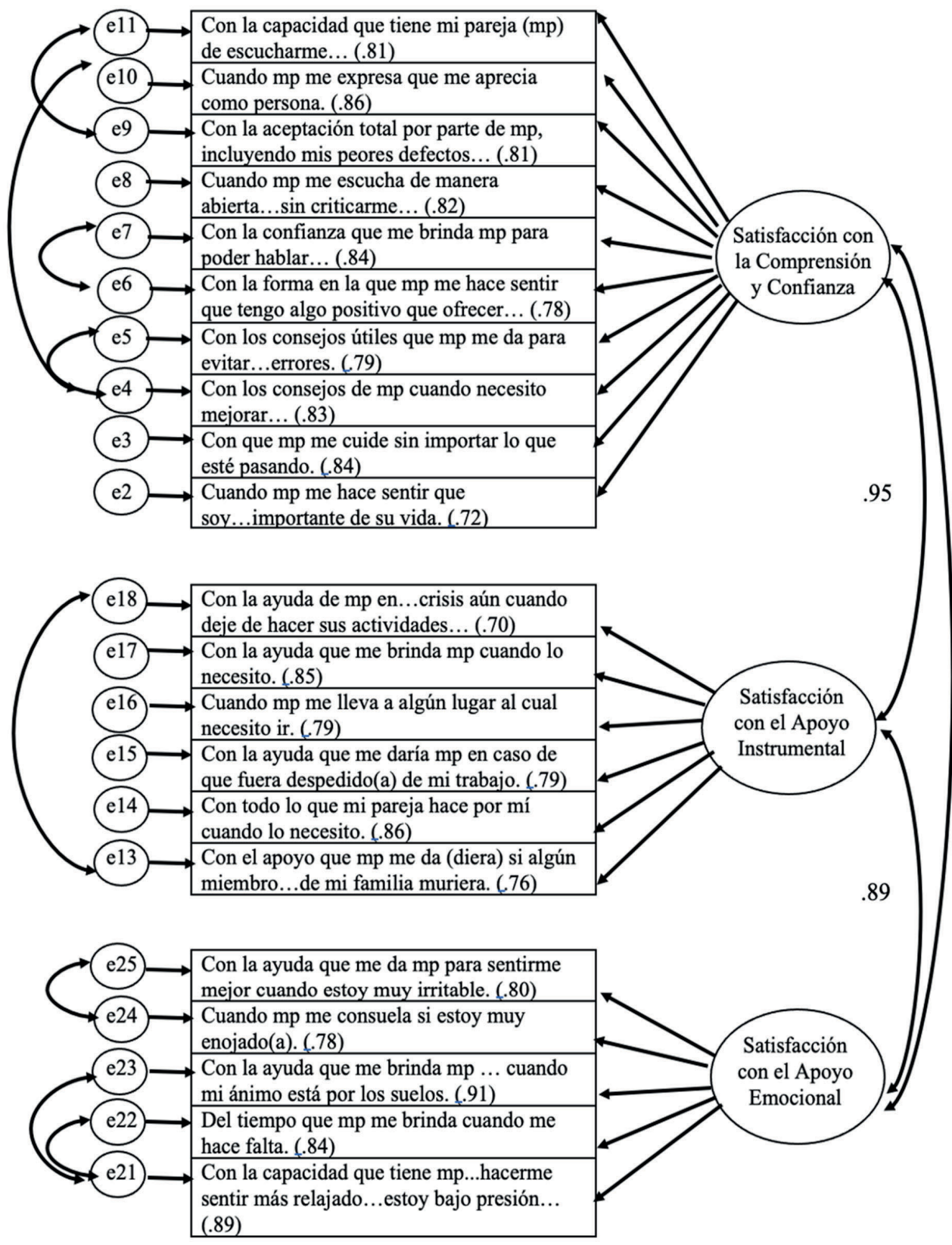




\section{R. SÁNCHEZ y N. CALLEJa}

Respecto a la EDaRAS, se realizó el mismo procedimiento estadístico (ver apartado Análisis de Datos) y se observó que los siete ítems diseñados discriminaron por lo que fueron sometidos a un análisis factorial de máxima verosimilitud con rotación oblicua (tipo promax) y se seleccionaron solo aquellos que registraron cargas factoriales iguales o superiores a .40. Dicho análisis reveló un índice $\mathrm{KMO}=.834$ y la Prueba de Esfericidad de Bartlett $=679.833, \mathrm{gl}=21, p=<.001$, además de indicar la existencia de un solo factor con valor propio superior a 1 que explicó el $35.94 \%$ de la varianza y con coeficientes de confiabilidad $\alpha=.791$ y $\omega=794$ (ver tabla 2).

Tabla 2

Análisis Factorial Exploratorio de la EDaRAS

\begin{tabular}{lcc}
\hline \multicolumn{1}{c}{ Reactivos } & Carga factorial \\
\hline Factor Disposición a Recibir Apoyo & .731 \\
Comparto mis sentimientos con otras personas para ver si me ayudan. & .662 \\
Dejo que las personas cercanas a mí me brinden su apoyo. & .648 \\
Hablo de mis problemas con los demás para abrir la posibilidad de recibir apoyo. & .615 \\
Permito que mi familia y amigos me apoyen cuando me encuentro en problemas. & .526 \\
Pido ayuda a otras personas cuando sé que no puedo hacer algo solo. & .509 \\
Estoy dispuesto a recibir consejos cuando me enfrento a una situación difícil. & .461 \\
Expreso mi sentir cuando algo me preocupa. & \\
\hline 7 reactivos & $\mathrm{M}=3.57$ \\
\hline
\end{tabular}

Acto seguido, se realizó el AFC en donde se obtuvo un modelo unidimensional (ver figura 2, se muestran las cargas factoriales estandarizadas), lo que confirma el ajuste del modelo y cuyos índices de ajuste obtenidos resultaron satisfactorios: $\chi^{2}(13)=19.062, \mathrm{CMIN} / \mathrm{DF}=1.466, \mathrm{NFI}=.972, \mathrm{CFI}=.991$, $\mathrm{AGFI}=.971, \mathrm{SRMR}=.026, \mathrm{RMSEA}=.034(.000-.065)$. 
Figura 2

Análisis Factorial Confirmatorio del modelo de un factor de la EDaRA

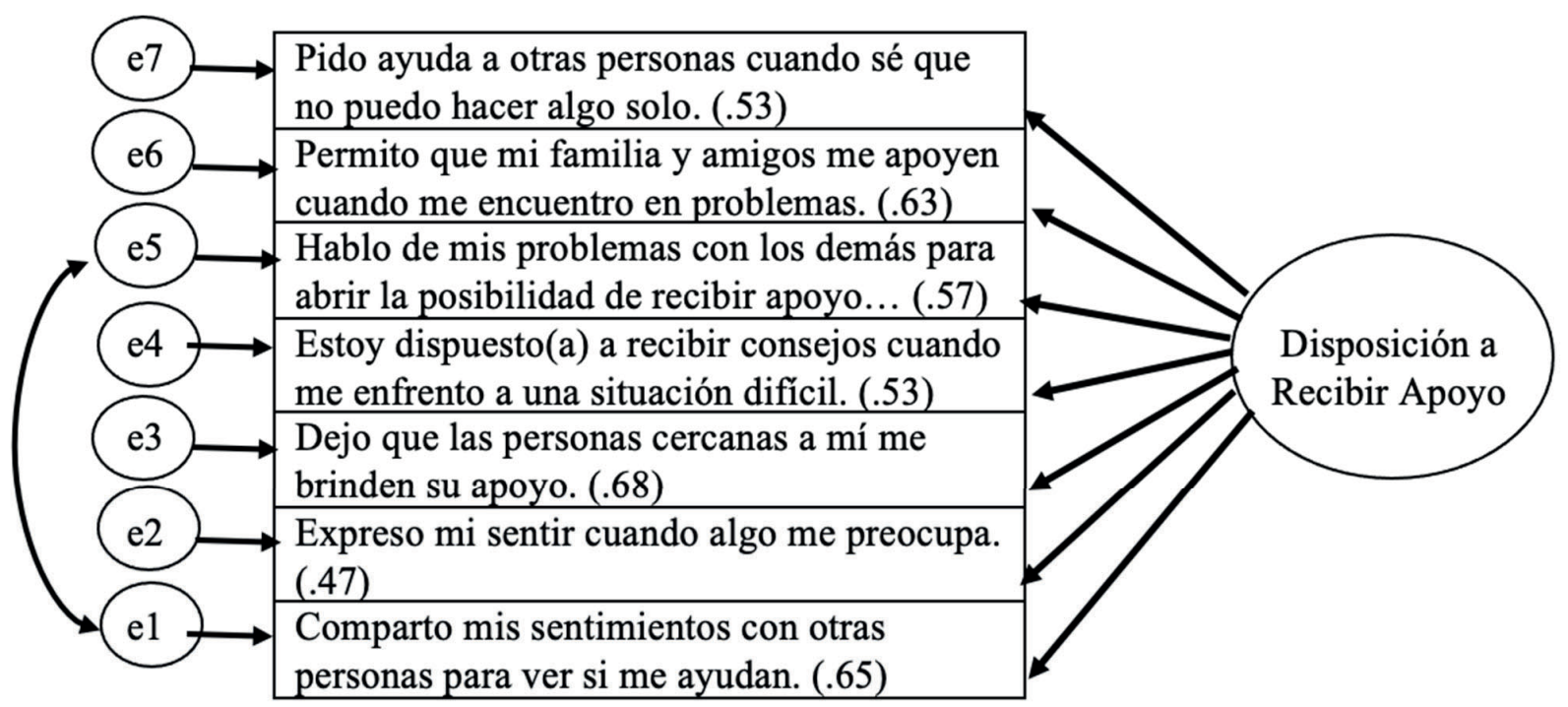

Se efectuaron AFC multi-grupo para probar los modelos de invarianza, que proponían que la ESAReP contendría una estructura de tres factores y la EDaRA sería unifactorial, tanto en hombres como en mujeres. En la tabla 3 se muestran los resultados de las pruebas de los modelos de invarianza de configuración, línea base o libre (M1), de invarianza métrica o débil (M2), de invarianza escalar o fuerte (M3) y de invarianza estricta (M4). Para la ESAReP, estos indicaron que cuando las cargas factoriales, interceptos y residuales de la estructura factorial se mantuvieron progresivamente invariantes en función del sexo, fueron comparables los índices de ajuste CFI y RMSEA; $\sin$ embargo, los $\Delta \chi^{2}$ resultaron significativos. En el caso de la EDaRA, se encontró invarianza métrica y estricta, pero faltó el parámetro de la invarianza escalar para obtener la equivalencia completa de la estructura de la escala por sexo (ver tabla 3). 
Tabla 3

Índices de ajuste para las pruebas de los modelos de Invarianza por sexo para la ESAReP y EDaRAS

$\begin{array}{llllcllll}\text { Modelo } & \chi^{2}(\mathrm{gl}) & \chi^{2} / \mathrm{gl} & \text { CFI } & \begin{array}{c}\text { RMSEA } \\ (\text { IC } 90 \%)\end{array} & \text { Comparación } & \Delta \chi^{2} & \Delta \text { CFI } & \Delta \text { RMSEA }\end{array}$

Escala de Satisfacción con el Apoyo Recibido de la Pareja

de configuración (356) (.045-.056)

(Línea base)

métrica o débil

$\begin{array}{lll}737.409 & 1.972 \quad .953\end{array}$

.049

M2 vs. M1

$19.981 \quad .000$

$-.001$

( $\lambda$ restringidas)

(374)

$(.044-.054)$

(18),

$p=.334$

M3. Invarianza

$\begin{array}{lll}780.545 & 1.976 \quad .995\end{array}$

.049

(.044-.054)

M3 vs. M2

43.136

.002

.000

escalar o

(395)

(21),

$p=.003$

restringidos)

M4. Invarianza

$\begin{array}{lll}891.703 & 2.074 \quad 993\end{array}$

.052

M4 vs. M3

111.158

$-.002$

.003

estricta $(\lambda, \tau, \theta$ y (430)

(.047-.056)

(35),

$p<.001$

Escala de Disposición a Recibir Apoyo Social

\begin{tabular}{|c|c|c|c|c|c|c|c|c|}
\hline $\begin{array}{l}\text { M1. Invarianza } \\
\text { de configuración } \\
\text { (Línea base) }\end{array}$ & $\begin{array}{c}34.382 \\
(26)\end{array}$ & 1.322 & .987 & $\begin{array}{c}.028 \\
(.000-.052)\end{array}$ & & & & \\
\hline $\begin{array}{l}\text { M2. Invarianza } \\
\text { métrica o débil } \\
(\lambda \text { restringidas) }\end{array}$ & $\begin{array}{c}38.091 \\
(32)\end{array}$ & 1.190 & .990 & $\begin{array}{c}.022 \\
(.000-.045)\end{array}$ & M2 vs. M1 & $\begin{array}{c}3.709(6) \\
p=.716\end{array}$ & .003 & -.006 \\
\hline $\begin{array}{l}\text { M3. Invarianza } \\
\text { escalar o } \\
\text { fuerte ( } \lambda \text { y } \tau \\
\text { restringidos) }\end{array}$ & $\begin{array}{c}59.506 \\
(39)\end{array}$ & 1.526 & .968 & $\begin{array}{c}.036 \\
(.015-.054)\end{array}$ & M3 vs. M2 & $\begin{array}{c}21.415(7) \\
p=.003\end{array}$ & -.022 & .014 \\
\hline $\begin{array}{l}\text { M4. Invarianza } \\
\text { estricta }(\lambda, \tau, \theta \text { y } \\
\text { restringidos) }\end{array}$ & $\begin{array}{c}70.942 \\
(48)\end{array}$ & 1.478 & .964 & $\begin{array}{c}.034 \\
(.015-.051)\end{array}$ & M4 Vs. M3 & $\begin{array}{c}11.436(9) \\
p=.247\end{array}$ & -.002 & -.002 \\
\hline Criterios de corte & & & & & & $p>.05$ & $p \leq .01$ & $p \leq .015$ \\
\hline
\end{tabular}

Nota. $\lambda$ : Cargas factoriales; $\tau$ : Interceptos; $\theta$ : Varianzas de error; CFI: Índice de Ajuste Comparativo; RMSEA: Raíz del Error Cuadrático Medio. 
Adaptación y VALIDACión de la Escala de Satisfacción CON El Apoyo Recibido de la Pareja

\section{Discusión}

La operacionalización del apoyo ha sido un tema recurrente en la literatura, en general, pero en menor medida al tratarse del apoyo entre los miembros de la pareja. Esto puede deberse a que sus componentes o indicadores se encuentran incluidos de manera implícita en medidas de afectividad, intimidad, conductas de interacción, entre otros, porque, como se señaló al inicio de este trabajo, el apoyo puede expresarse de muchas maneras, lo cual tiene implicaciones significativas en dichos constructos (Guerrero et al., 2018). Esto es justamente lo que impide que se estudie particularmente y se identifiquen sus matices y relaciones con otras variables inseparables de la vida de la pareja. Sin embargo, este tema ha recibido contribuciones interesantes que han buscado el operacionalizar los roles inmersos en el constructo (recibir y dar apoyo), la diferencia entre lo esperado con lo recibido, e incluso sus tipos (emocional o instrumental); no obstante, el hablar de su frecuencia o acuerdo no satisfacen o previenen aspectos como recibir apoyo en el tiempo y contenido requeridos, y que el proceso de recepción así como la reciprocidad sean cubiertas. De acuerdo con Rafaeli y Gleason (2009), todos estos aspectos impactan en que realmente el apoyo sea exitoso entre los miembros de una diada. Por ello, en esta investigación se tomó como base al Cuestionario de Apoyo Social (Sarason et al., 1983), que conceptualmente es claro, aborda una buena parte de los escenarios en los que el apoyo puede ser necesario en una pareja y es uno de los más utilizados (Martínez-López et al. 2014). Así, se realizó el proceso de traducción-retraducción-adaptación, se agregaron algunos ítems y se puso a prueba con parejas mexicanas.

En cuanto a los dos primeros objetivos, se obtuvieron dos escalas válidas y confiables para medir la satisfacción con el apoyo recibido y la disposición de recibirlo, porque cumplieron con los lineamientos de congruencia conceptual y de confiabilidad robustos (Reyes-Lagunes \& García-y-Barragán, 2008).

Con respecto a la Escala de Satisfacción con el Apoyo Recibido de la Pareja (ESAReP), los AFE y AFC mostraron la existencia de tres factores cuyos índices resultaron satisfactorios. El primero se denominó Satisfacción con la Comprensión y Confianza, pues versa sobre cuánto sienten las personas la complacencia ante la capacidad de escucha abierta y sin crítica y el consejo que la pareja provee, así como con el aprecio y el valor, la aceptación, la confianza, el cuidado y la importancia que la pareja le da al participante. Al respecto, Fiske (1992) y Vangelisti (2009) señalan que el apoyo implica conductas de compartir, porque implica la comunicación evidente en la capacidad de escuchar y dar consejos con el fin de enriquecer a la pareja, lo que permite crear un sentido de comprensión y balance en las interacciones constantes de la pareja. Este factor muestra cómo la persona realiza comportamientos de mantenimiento día tras día en la relación que le hace sentir al otro miembro de la pareja comprendido, respaldado, valioso (Procidano \& Walker-Smith, 1997; Sánchez-Aragón \& Díaz-Loving, 2002) apreciado y amado (Sánchez-Aragón, 2018) y con potencial para reforzar su capacidad de resistir y superar frustraciones (Chi et al., 2011), resolver retos (NSCDC, 2010) al contribuir con su ajuste psicológico y salud física (Sarason et al., 1983). Además, puede resultar no solo en el bienestar individual y relacional (VidalGonzález et al., 2012), sino también en un indicador relevante de compromiso en la relación de pareja (Weigel \& Ballard-Reisch, 2014).

El segundo factor llamado Satisfacción con el Apoyo Instrumental evalúa el gusto que las personas participantes sienten respecto al soporte y la ayuda tangible que reciben en caso de necesidad (e.g., llevar a 


\section{R. SÁNCHez y N. CALLEJa}

algún lugar) o recibirían si se les presentaran situaciones de la vida más difíciles, delicadas y serias como una crisis o duelo; en las cuales quien brinda cuidado, sacrifica parte de su vida (dinero, esfuerzo) al evidenciar con ello la importancia que su pareja tiene para él o ella (Cutrona \& Russell, 1987; Sarason et al., 1983). Se le denomina instrumental pues se refiere, de acuerdo con Morelli et al. (2015), a la ayuda o la asistencia de una manera tangible o física tal como proveer dinero, dar soluciones prácticas o colaborar realizando una tarea.

Por su parte, el tercer factor nombrado Satisfacción con el Apoyo Emocional, se trata del gozo experimentado cuando la pareja amortigua, consuela, relaja cuando la persona participante se siente irritable, con enojo y bajo presión; además de que le levanta el ánimo cuando está disminuido. Al respecto, desde la perspectiva de Morelli et al. (2015) el apoyo emocional involucra escuchar, ser empático y ofrecer afecto ante eventos de necesidad, lo cual se aprecia en las conductas que reconfortan a la pareja cuando experimenta estrés, o bien decaimiento. Asimismo, este refleja, a su vez, que cuando el proveedor de apoyo está siendo empático (Devoldre et al., 2010; Verhofstadt et al., 2016) con su pareja, además de mostrar que cuenta con un estado emocional positivo, se enfoca en su relación seguramente al ser optimista y mediante buen manejo emocional (Barbee et al., 1998).

Como es evidente, los contenidos de los factores obtenidos constatan el hallazgo de correlaciones altas reportadas en la figura 1, porque la satisfacción con la comprensión y la confianza que siente la persona participante (quien recibe el apoyo) fortalece la aceptación y el valor dentro de su relación para su pareja (Guerrero et al., 2018). Ello le permite gozar del apoyo instrumental y emocional, que se contribuyen mutuamente; es decir, en la medida que se siente satisfacción con un aspecto se tiene con los otros aspectos. Aunado a esto, los factores derivados muestran "tonalidades" del constructo en el contexto de la relación de pareja, que si bien no aparecían en la versión original de Sarason et al. (1983), ni en la escala mexicana de Cienfuegos-Martínez y Díaz-Loving (2011), porque ambas son escalas unidimensionales-, aquí están bien definidas y muestran cualidades psicométricas aceptables.

Pero, la satisfacción con el apoyo recibido por parte de la pareja posee un precedente individual que se volvió central operacionalizar: la capacidad que tenían los participantes de recibir apoyo. Esto, principalmente, porque, por un lado, implica la línea base para sentir la satisfacción con el apoyo recibido y, por otro lado, que en la revisión de literatura no se encontró algo parecido a ello ni en términos conceptuales ni en operacionales. Así, la Escala de Disposición a Recibir Apoyo Social (EDaRAS) mide la inclinación de las personas de compartir sus sentimientos, abrir la posibilidad de recibir apoyo y consejos, aceptar la ayuda brindada por familia y amigos en momentos de necesidad, a la vez que reconocer la falta de recursos para solucionar los problemas de manera individual. Este factor expresa tanto el reconocimiento personal de la falta de capacidad para enfrentar la amenaza inherente a un evento desencadenador de estrés (Cohen, 2004) como la percepción de fuentes posibles de apoyo como pueden ser otros significativos -entre ellos la pareja- que pueden proveer de ayuda, cuidado y atención, particularmente en momentos de dificultad. En congruencia, Eisenberger (2013) encontró que dejarse ayudar y/o recibir apoyo puede beneficiar la salud a través de la activación de regiones neurales que responden a la seguridad e inhiben las respuestas fisiológicas relacionadas a la amenaza.

Lo anterior evidencia la conexión inseparable entre el apoyo social y la disposición a recibir apoyo, porque esta última facilita la apertura hacia la obtención de cuidado, amor, responsividad emocional, 
Adaptación y validación de la Escala de Satisfacción con el Apoyo Recibido de la Pareja

consistencia y confiabilidad (NSCDC, 2010). Dichos elementos funcionan en conjunto como un factor protector de cada miembro de la pareja (Procidano \& Walker-Smith, 2009; Southwick et al., 2016; Werner, 2000) y otorga una visión más completa del fenómeno bajo estudio.

Respecto al tercer objetivo que analizó la invarianza factorial de las escalas, al evaluar el grado de igualdad de su estructura entre hombres y mujeres, se encontró que, de acuerdo con los criterios recomendados por Cheung y Rensvold (2002), en la ESAReP los modelos puestos a prueba fueron comparables cuando las cargas factoriales se mantenían invariantes a través del sexo, pero cumplieron solo dos de los tres criterios en los modelos de invarianza escalar y estricta. En relación con la invarianza para la EDaRA, las pruebas efectuadas mostraron equivalencia de la estructura factorial, excepto en dos de los parámetros del modelo de invarianza escalar. Por tanto, en ambas escalas se asumiría una invarianza métrica parcial (Dimitrov, 2010), la cual sugiere que los puntajes de las escalas podrían ser equivalentes en ambos grupos. Estos resultados se apoyan en la investigación de Neff y Karney (2005), donde refieren similitud entre los miembros de la pareja al respecto del apoyo intercambiado entre ellos. Los estudios que reportan diferencias son respecto a la recepción, la solicitud y el estilo de apoyo (ver Belle, 1982; Pasch et al., 1997; Tannen, 1990; Thoits, 2011; Verhofstadt et al., 2007), no respecto a la satisfacción ante el apoyo recibido de la pareja. Dicho tema se aborda en esta investigación.

Con base en lo planteado, los instrumentos validados en esta investigación aportan una contribución metodológica al área de las relaciones personales, particularmente, a las de pareja, pues evalúan aspectos del apoyo emocional no estudiados con anterioridad en el contexto de la pareja y en México. Asimismo, el contenido proporciona conocimiento sobre las especificidades del apoyo recibido (satisfacción con la comprensión y confianza, con el apoyo instrumental y el emocional y la disposición a recibir apoyo) por parte de la pareja, brindan un mejor entendimiento del constructo. En cuanto a las limitaciones, se observó la escasa información o inespecificidad de la definición de la disposición para recibir apoyo social (ver Gurung et al., 1997) como una característica personal del receptor, lo cual podría examinarse más de cerca al identificar rasgos de personalidad que puedan favorecer dicha disposición y poder crear un perfil más claro que facilite o no la recepción de apoyo. Se sugiere continuar el trabajo en esta línea de investigación mediante la integración de variables que fortalezcan el entendimiento del funcionamiento del apoyo en este y otros contextos relacionales como lo pueden ser la resiliencia, la empatía y la regulación emocional en quien brinda el apoyo -entre otras-; asimismo -y de acuerdo con Verhofstadt et al. (2013) - se vuelve central en la práctica profesional, evaluar las conductas de apoyo en las parejas, explicarles el ciclo interactivo de conductas de apoyo, incrementar las habilidades de apoyo entre la pareja y detectar y bloquear la conducta de apoyo negativo al seguir las pautas planteadas por Rafaeli y Gleason (2009).

\section{Referencias}

Asociación Americana de Psicología (2020). Manual de publicaciones de la American Psychological Association (4ta ed). Manual Moderno. 


\section{R. SÁNCHEZ y N. CALLEJa}

Barbee, A. P., Rowatt, T. L., \& Cunningham, M. R. (1998). When a friend is in need: feelings about seeking, giving, and receiving social support. En P. A. Andersen \& L. K. Guerrero (Eds.), Handbook of Communication and Emotion (pp. 281-301). Academic Press. https://doi.org/10.1016/B978-012057770-5/50012-6

Belle, D. (1982). The stress of caring: women as providers of social support. En L. Goldberger \& S. Breznitz (Eds.), Handbook of stress: Theoretical and clinical aspects (pp. 496-505). Free Press.

Bodenmann, G. (2005). Dyadic coping and its significant for marital functioning. En T. Revenson, K. Kayser, \& G. Bodenmann (Eds.), Couples coping with stress: Emerging perspectives on dyadic coping (pp. 33-50). American Psychological Association. https://psycnet.apa.org/doi/10.1037/11031-002

Bodenmann, G., Plancherel, B., Beach, S. R. H., Widmer, K., Gabriel, B. Meuwly, N., ... Schramm, E. (2008). Effects of Coping-Oriented Couples Therapy on Depression: A Radomized Clinical Trial. Journal of Consulting and Clinical Psychology, 76(6), 944-954. https://doi.org/10.1037/a0013467

Bradbury, T. N., \& Pasch, L. A. (1994). The Social Support Interaction Coding System (SSICS). Unpublished coding manual. University of California.

Burkert, S., Knoll, N., \& Scholz, U. (2005). Korrelate der Rauchgewohnheiten von Studierenden und jungen Akademikern: Das Konzept der dyadischen Planung. Psychomed, 17, 240-246.

Chi, P., Tsang, S. K. M., Chan, K. S., Xiang, X., Yip, P. S. F., Cheung, Y. T., \& Zhang, X. (2011). Marital satisfaction of Chinese under stress: Moderating effects of personal control and social support. Asian Journal of Social Psychology, 14(1), 15-25. https://doi.org/10.1111/j.1467-839X.2010.01322.x

Cheung, G. W., \& Rensvold, R. B. (2002). Evaluating goodness-of-fit indexes for testing measurement invariance. Structural Equation Modeling, 9(2), 233-255. https://doi.org/10.1207/S15328007SEM0902 5

Cienfuegos-Martínez, Y. I., \& Díaz-Loving, R. (2011). Red social y apoyo percibido de la pareja y otros significativos: una validación psicométrica. Enseñanza e Investigación en Psicología, 16(1), 27-39.

Cohen, S. (2004). Social relationships and health. American Psychologist, 59, 676-684. https://psycnet.apa.org/ doi/10.1037/0003-066X.59.8.676

Collins, N. L., \& Feeney, B. C. (2010). An attachment theoretical perspective on social support dynamics in couples: Normative processes and individual differences. En K. Sullivan \& J. Davila (Eds.), Support Processes in Intimate Relationships (pp. 89-120). Oxford University Press. https://doi.org/10.1002/9781118001868.ch13

Cronbach,L.J., \& Meehl,P.E.(1955). Constructvalidity in psychological tests. Psychological Bulletin, 52(4), 281-302.

Cutrona, C. E., \& Russell, D. W. (1987). The Provisions of Social Relationships and Adaptation to Stress. En W. H. Jones \& D. Perlman (Eds.), Advances in Personal Relationships (Vol. 1, pp. 37-67). JAI Press.

Dainton, M. (2011). Linking Theoretical Explanations for the Use of Marital Maintenance: Equity, Uncertainty, Attachment, and Reciprocity. Acta de Investigación Psicológica, 1(2), 352-374. http://www.scielo.org.mx/ pdf/aip/v1n2/v1n2a10.pdf

Dehle, C., Larsen, D., \& Landers, J. E. (2001). Social Support in Marriage. American Journal of Family Therapy, 29(4), 307-324. https://psycnet.apa.org/doi/10.1080/01926180152588725

Devoldre, I., Davis, M. H., Verhofstadt, L. L., \& Buysse, A. (2010). Empathy and social support provision in couples: social support and the need to study the underlying process. The Journal of Psychology, 144(3), 259-284. https://doi.org/10.1080/00223981003648294

Dimitrov, D. M. (2010). Testing for factorial invariance in the context of construct validation. Measurement and Evaluation in Counseling and Development, 43(2), 121-149. https://doi.org/10.1177/0748175610373459 
Adaptación y validación de la Escala de Satisfacción con el Apoyo Recibido de la Pareja

Eisenberger, N. I. (2013). An Empirical Review of the Neural Underpinnings of Receiving and Giving Social Support: Implications for Health. Psychosomatic Medicine, 75(6), 545-556. https://doi.org/10.1097/ PSY.0b013e31829de2e7

Falconier, M. K., \& Kuhn, R. (2019). Dyadic Coping in Couples: A Conceptual Integration and a Review of the Empirical Literature. Frontier in Psychology, 10, 1-23. https://doi.org/10.3389/fpsyg.2019.00571

Fiske, A. P. (1992). The four elementary forms of sociality: framework for a unified theory of social relations. Psychology Review, 99(4), 689-723. https://psycnet.apa.org/doi/10.1037/0033-295X.99.4.689

Flórez-Rodríguez, N., \& Sánchez-Aragón, R. (2019). El Estrés (experiencia y percepción de reto vs amenaza) y la Rumia como factores de Riesgo a la Salud. Revista Salud y Administración, 7(20), 17-27.

Gable, S. L., Gonzaga, G. C., \& Strachman, A. (2006). Will you be there for me when things go right? Supportive responses to positive event disclosures. Journal of Personality and Social Psychology, 91(5), 904-917. https://psycnet.apa.org/doi/10.1037/0022-3514.91.5.904

Gómez-Zapiain, J., Ortiz, M. J., \& Gómez-Lope, J. (2012). Capacidad para aportar y solicitar apoyo emocional en las relaciones de pareja en relación con los perfiles de apego. Anales de Psicología, 28(1), 302-312.

Greenberg, L., \& Johnson, S. M. (1988). Emotionally Focused Therapy for Couples. The Guilford Press.

Guerrero, L. K., Andersen, P A., \& Afifi, W. A. (2018). Close Encounters: Communication in Relationships. Sage Publications, Inc.

Gurung, R.A.R., Sarason, B., \& Sarason, I. (1997). Personal characteristics, relationships quality, and social support perceptions and behavior in young adult romantic relationships. Personal Relationships, 4(4), 319-339.

Hu, L., \& Bentler, P. M. (1999). Cutoff criteria for fit indexes in covariance structure analysis: Conventional criteria versus new alternatives. Structural Equation Modeling, 6(1), 1-55. https://doi. org/10.1080/10705519909540118.

Karney, B. R., Story, L. B., \& Bradbury, T. N. (2005). Marriages in context: Interactions between chronic and acute stress among newlyweds. En T. A. Revenson, K. Kayser, \& G. Bodenmann (Eds.), Couples coping with stress: Emerging perspectives on dyadic coping (pp. 13-32). American Psychological Association.

Martínez-López, Z., Páramo-Fernández, M.F., Guisande-Couñago, M. A., Tinajero-Vacas, C., da Silva-Almeida, L., \& Rodríguez-González, M. S. (2014). Apoyo social en universitarios españoles de primer año: propiedades psicométricas del Social Support Questionnaire-Short Form y el Social Provisions Scale. Revista Latinoamericana de Psicología, 46(2), 102-110. https://doi.org/10.1016/S0120-0534(14)70013-5

Morelli, S. A., Lee, I. A., Arnn, M. E., \& Zaki, J. (2015). Emotional and instrumental support provision interact to predict well-being. Emotion, 15(4), 484-493. https://doi.apa.org/doi/10.1037/emo0000084

National Scientific Council on the Developing Child (2010). Early experiences can alter gene expression and affect long-term development. Working paper no. 10. https://developingchild.harvard.edu/wp-content/ uploads/2010/05/Early-Experiences-Can-Alter-Gene-Expression-and-Affect-Long-Term-Development.pdf

Neff, L. A., \& Karney, B. R. (2005). Gender Differences in Social Support: A Question of Skill or Responsiveness? Journal of Personality and Social Psychology, 88(1), 79-90. https://doi.org/10.1037/0022-3514.88.1.79

Palomar-Lever, J., \& Gómez-Valdez, N. E. (2010). Desarrollo de una escala de medición de la resiliencia con mexicanos (RESI-M). Interdisciplinaria, 27(1), 7-22.

Pasch, L. A., Bradbury, T., \& Davila, J. (1997). Gender, negative affectivity, and observed social support behavior in marital interaction. Personal Relationships, 4(4), 361-378. 


\section{R. SÁNCHez y N. CALLeja}

Procidano, M. E., \& Walker-Smith, W. (1997). Assessing perceived social support: the importance of context. En G. R. Pierce, B. Lakey, I. G. Sarason, \& B. R. Sarason (Eds.). Sourcebook of Social Support and Personality (pp. 93-106). Plenum Press. https://doi.org/10.1007/978-1-4899-1843-7_5

Rafaeli, E., \& Gleason, M. E. J. (2009). Skilled Support within Intimate Relationships. Journal of Family Theory \& Review, 1(1), 20-37. https://doi.org/10.1111/j.1756-2589.2009.00003.x

Revenson, T. A. (1994). Social support and marital coping with chronic illness. Annals of Behavioral Medicine, $16(2), 122-130$.

Reyes-Lagunes, I., \& García-y-Barragán, L. F. (2008). Hacia un procedimiento de validación psicométrica culturalmente relevante. En S. Rivera-Aragón, R. Díaz-Loving, R. Sánchez-Aragón \& I. Reyes-Lagunes (Eds.), La Psicología Social en México XII (pp. 625-630). AMEPSO.

Rivas-Diez, R. (2013). Apoyo Social Funcional en mujeres de la población general y en mujeres maltratadas chilenas. Propiedades psicométricas del Duke-UNC-11. Revista Iberoamericana de Diagnóstico y Evaluación Psicológica, 36(2), 9-27.

Sánchez-Aragón, R. (2016). La Pareja Romántica en México: Desentrañando su Naturaleza Comunicativa y Emocional. Universidad Nacional Autónoma de México.

Sánchez-Aragón, R. (2018). Afectividad y Co-Regulación en la Pareja: Validación de un Inventario y Variaciones a través del Ciclo Vital. Revista Psicologìa e Eduçao (On-Line), 1(1), 58-69.

Sánchez-Aragón, R., \& Díaz-Loving, R. (2002). De lo aceptable a lo ofensivo en la relación de pareja. Revista de Psicología Social y Personalidad, XVIII (2), 55-75.

Sarason, I. G., Levine, H. M., Basham, R. B., \& Sarason, B. R. (1983). Assessing Social Support: The Social Support Questionnaire. Journal of Personality and Social Psychology, 44(1), 127-139.

Secretaría de Salud (2021). Ley General de Salud. http://www.diputados.gob.mx/LeyesBiblio/pdf mov/Ley General_de_Salud.pdf

Southwick, S.M., Sipper, L., Krystal, J., Charney, D., Mayes, L., \& Pietrzak, R. H. (2016). Why are some individuals more resilient than others? the role of social support. World Psychiatry, 15(1), 77-79. https://doi. org/10.1002/wps.20282

Sprecher, S. \& Felmlee, D. (1992). The influence of parents and friends on the quality and stability of romantic relationships: A three-wave longitudinal investigation. Journal of Marriage and the Family, 54(4), 888-900. https://psycnet.apa.org/doi/10.2307/353170

Stafford, L. (2003). Maintaining romantic relationships: A summary and analysis of one research program. En D. J. Canary \& M. Dainton(Eds.), Maintaining relationships through communication (pp. 59-78). Lawrence Erlbaum.

Sociedad Mexicana de Psicología (2009). Código ético del psicólogo. Trillas.

Sullivan, K. T., \& Davila, J. (2010). Introduction. En K. Sullivan \& J. Davila (Eds.), Support processes in intimate relationships (pp. xix-xxx). Oxford University Press.

Tannen, D. (1990). You just don't understand. Ballantine Books.

Thoits, P. A. (2011). Mechanisms linking social ties and support to physical and mental health. Journal of Health and Social Behavior, 52(2), 145-161. https://doi.org/10.1177\%2F0022146510395592

Vandenberg, R., \& Lance, C. (2000). A review and synthesis of the measurement invariance literature: Suggestions, practices, and recommendations for organizational research. Organizational Research Methods, 3(1), 4-70. https://doi.org/10.1177/109442810031002 
Adaptación y Validación de la Escala de Satisfacción con el Apoyo Recibido de la Pareja

Vangelisti, A. L. (2009). Challenges in conceptualizing social support. Journal of Social and Personal Relationships, 26(1), 39-51. https://doi.org/10.1177\%2F0265407509105520

Verhofstadt, L.L., Buysse, A., \& Ickes, W. (2007). Social Support in Couples: An Examination of Gender Differences Using Self-report and Observational Methods. Sex Roles 57, 267-282. https://doi.org/10.1007/ $\underline{\text { s11199-007-9257-6 }}$

Verhofstadt, L. L., Devoldre, I., Buysse, A., Stevens, M., Hinnekens, C., Ickes, W., \& Davis, M. (2016) The Role of Cognitive and Affective Empathy in Spouses' Support Interactions: An Observational Study. PLoS ONE 11(2): e0149944. https://doi.org/10.1371/journal.pone.0149944

Verhofstadt, L. L., Lemmens, G., \& Buysse, A. (2013). Support-seeking, support-provision and support-perception in distressed married couples: a multi-method analysis. Journal of Family Therapy, 35(3), 320-339. https:// doi.org/10.1111/1467-6427.12001

Verhofstadt, L. L., \& Weytens, F. (2013). Biological sex and gender role identity as predictor of spousal support provision: a scenario-based study. Journal of Gender Studies, 22(2), 166-177. https://doi.org/10.1080/0958 $\underline{9236.2012 .745683}$

Vidal-González, L. F., Rivera-Aragón, S., Díaz-Loving, R., \& Méndez-Ramírez, I. (2012). Elaboración de una escala de permanencia en la relación de pareja. Revista Iberoamericana de Diagnóstico y Evaluación Psicológica, 1(33), 199-225.

Weigel, D. J. \& Ballard-Reisch, D. S. (2014). Constructing commitment in intimate relationships: mapping interdependence in the everyday expressions of commitment. Communication Research, 41(3), 311-332. https://doi.org/10.1177\%2F0093650212440445

Werner, E. E. (2000). Protective factors and individual resilience. En J. P. Shonkoff \& S. J. Meisels (Eds.), Handbook of Early Childhood Intervention (pp. 115-134). Cambridge University Press. https://doi.org/10.1017/ CBO9780511529320.008

Yárnoz-Yaben, S. (2010). Cuestionario de Apoyo Recibido de la Ex Pareja (CARE): un Instrumento Breve para Evaluar la Co-Parentalidad Post Divorcio. Revista de Psicopatología y Psicología Clínica, 15(2), 133-142. https://doi.org/10.5944/rppc.vol.15.num.2.2010.4092

Zimet, G. D., Dahlem, N. W., Zimet, S. G., \& Farley, G. K. (1988). The Multidimensional Scale of Perceived Social Support. Journal of Personality Assessment, 52(1), 30-41. https://doi.org/10.1207/s15327752jpa5201_2

Recibido: 6 de junio de 2019 Revisión recibida: 16 de marzo de 2021

Aceptado: 13 de mayo de 2021 


\section{Sobre las autoras}

Rozzana Sánchez Aragón es doctora en Psicología Social por la Universidad Nacional Autónoma de México (México), en donde se desempeña como profesora de tiempo completo desde hace 26 años. Es miembro del Sistema Nacional de Investigadores y ha dirigido 11 proyectos financiados, ha publicado 98 artículos de investigación, 143 capítulos de libros y seis libros sobre sus áreas de especialidad. Asimismo, ha presentado 671 ponencias y 70 conferencias magistrales en congresos nacionales e internacionales.

Nazira Calleja es Doctora en Psicología en la Facultad de Psicología de la Universidad Nacional Autónoma de México. Actualmente, labora en esa institución y se dedica a la actividad académica: imparte clases de metodología, estadística básica e intermedia y construcción de instrumentos, dirige tesis, imparte conferencias y publica artículos en revistas especializadas. Compiló el libro "Psicología Social: Teoría e Investigación en México" y los inventarios electrónicos "Escalas Psicosociales en México" y "Catálogo de Escalas Psicosociales y de la Salud en Español”, los cuales se alojan en la página web de la Facultad de Psicología de la UNAM. En los últimos años, se ha dedicado a la investigación en comportamiento tabáquico en mujeres adolescentes y al estudio, construcción y evaluación psicométrica de escalas psicosociales.

Publicado en línea: 30 de junio de 2021 


\section{Apéndice}

\section{Escala de Disposición a Recibir Apoyo Social (EDaRAS)}

INSTRUCCIONES. A continuación, se presenta una serie de conductas o disposiciones que las personas hacemos cuando enfrentamos una situación que puede rebasar nuestras capacidades. Por favor, indique con una $\mathrm{X}$ con qué frecuencia usted realiza lo que se le menciona.

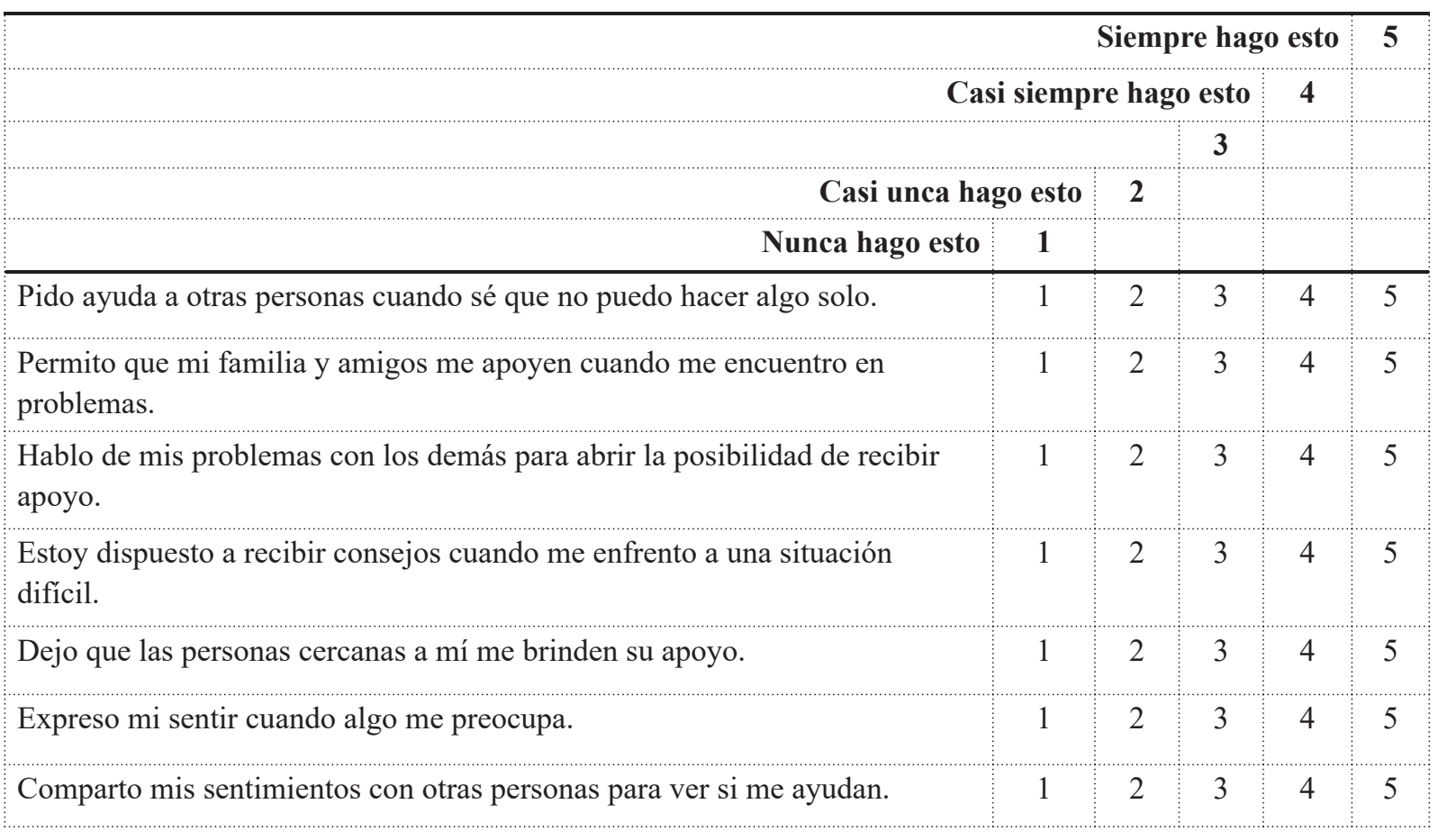

